\title{
On the density of extremal solutions of differential inclusions
}

\author{
by F. S. De Blasi (Roma) and G. Pianigiani (Siena)
}

\begin{abstract}
An existence theorem for the Cauchy problem $(*) \dot{x} \in \operatorname{ext} F(t, x), x\left(t_{0}\right)=$ $x_{0}$, in Banach spaces is proved, under assumptions which exclude compactness. Moreover, a type of density of the solution set of $(*)$ in the solution set of $\dot{x} \in F(t, x), x\left(t_{0}\right)=x_{0}$, is established. The results are obtained by using an improved version of the Baire category method developed in [8]-[10].
\end{abstract}

1. Introduction. Let $\mathbb{E}$ be a separable reflexive real Banach space. Let $F$ be a continuous multifunction defined on a nonempty open subset of $\mathbb{R} \times \mathbb{E}$ with values in the space of closed convex bounded subsets of $\mathbb{E}$ with nonempty interior. We shall consider the Cauchy problems

$$
\begin{gathered}
\dot{x} \in F(t, x), \quad x\left(t_{0}\right)=x_{0}, \\
\dot{x} \in \operatorname{ext} F(t, x), \quad x\left(t_{0}\right)=x_{0},
\end{gathered}
$$

where ext $F(t, x)$ denotes the set of extreme points of $F(t, x)$.

By a result of Pliś ([2], p. 127) the solution set $\mathcal{M}_{\text {ext } F}$ of (1.2) is not, in general, dense in the solution set $\mathcal{M}_{F}$ of (1.1). Nevertheless, elements of $\mathcal{M}_{\text {ext } F}$ do approximate some significant subsets of $\mathcal{M}_{F}$. More specifically, we shall prove that, for any selection $f$ of $F$ in an admissible class which includes locally $\alpha$-Lipschitz selections, if we denote by $K_{f}$ the solution set of the Cauchy problem

$$
\dot{x}=f(t, x), \quad x\left(t_{0}\right)=x_{0},
$$

then $\mathcal{M}_{\text {ext } F}$ has nonempty intersection with every neighborhood of $K_{f}$. In particular, the Cauchy problem (1.2) has solutions.

In finite dimensions this type of approximation result has been established by Pianigiani [16], by using the technique of Antosiewicz and Cellina [1]. Additional difficulties occur in infinite dimensions because, in this setting, the existence theory for differential equations is more delicate [12].

1991 Mathematics Subject Classification: 34A60, 47H05. 
For recent contributions, see Tolstonogov [17], Bahi [3], Tolstonogov and Finogenko [18], Papageorgiou [14], [15].

The approach used in the present paper is a variant of the Baire category method introduced in [8]-[10] in order to prove the existence of solutions for nonconvex-valued differential inclusions in Banach spaces. We mention that recently this method has been improved by Bressan and Colombo [4], who have obtained an existence theorem containing both the existence theorem of [10] and Filippov's theorem [11] (see also Kaczyński and Olech [13], Antosiewicz and Cellina [1]). The property that $\mathcal{M}_{\operatorname{ext} F} \neq \emptyset$ has been proved in [10], under stronger hypotheses; subsequently the same result has been established in [7], by following the method and the techniques of [10].

2. Preliminaries and auxiliary results. Let $\mathbb{E}$ be a reflexive separable real Banach space with norm $\|\cdot\|$. We denote by $\mathcal{B}$ the metric space of all closed convex bounded subsets of $\mathbb{E}$, with nonempty interior, endowed with the Hausdorff distance $h$.

Let $Z$ be a metric space. A multifunction $G: Z \rightarrow \mathcal{B}$ is said to be continuous, bounded, if it so as a function from $Z$ to the metric space $\mathcal{B}$. Let $X$ be a nonempty subset of $Z$. A single-valued function $f: X \rightarrow \mathbb{E}$ satisfying $f(x) \in G(x)$ for every $x \in X$ is called a selection of $G$ on $X$ (a selection of $G$ if $X=Z$ ). For any subset $X$ of $Z$, the interior of $X$ and the closure of $X$ are denoted by int $X$ and $\bar{X}$, respectively. Moreover, if $X \subset Z$ is bounded, $\alpha[X]$ stands for the Kuratowski measure of noncompactness of $X$. In $Z$ an open (resp. closed) ball with center $x \in Z$ and radius $r>0$ is denoted by $B(x, r)$ (resp. $\widetilde{B}(x, r))$. The unit open ball in a normed space $Z$ is denoted by $B$; moreover, for any subset $X$ of $Z$, ext $X$ stands for the set of extreme points of $X$.

Let $J$ be a nonempty bounded interval of $\mathbb{R}$. As usual, $C(J, \mathbb{E})$ denotes the Banach space of all continuous bounded functions $x: J \rightarrow \mathbb{E}$ endowed with the norm of uniform convergence. Furthermore, by $|J|$ we mean the length of $J$. The space $\mathbb{R} \times \mathbb{E}$ will be equipped with the norm $\|(t, x)\|=$ $\max \{|t|,\|x\|\},(t, x) \in \mathbb{R} \times \mathbb{E}$. In the sequel, when a set $X \subset Z$ is considered as a metric space, it is understood that $X$ retains the metric of $Z$.

Let $U$ be a nonempty subset of $\mathbb{R} \times \mathbb{E}$. A function $f: U \rightarrow \mathbb{E}$ is said to be $\alpha$-Lipschitzean (with constant $k$ ) if $f$ is continuous and bounded on $U$, and there exists a constant $k \geq 0$ such that $\alpha[f(X)] \leq k \alpha[X]$ for every bounded set $X \subset U$. A function $f: U \rightarrow \mathbb{E}$ is said to be locally Lipschitzean (resp. locally $\alpha$-Lipschitzean) if $f$ is bounded (resp. continuous and bounded), and for each $(s, u) \in U$ there exist $\delta_{s, u}>0$ and $k_{s, u} \geq 0$ such that $f$ restricted to $B\left((s, u), \delta_{s, u}\right)$ is Lipschitzean (resp. $\alpha$-Lipschitzean) with constant $k_{s, u}$.

Let $J$ be a nonempty bounded interval of the form $[a, b[$. We denote by $\mathcal{I}(J)$ the class of all countable families $\left\{J_{i}\right\}$ of nonempty pairwise disjoint 
intervals $J_{i}=\left[a_{i}, b_{i}\left[\right.\right.$ such that $\bigcup_{i} J_{i}=J$. A member of $\mathcal{I}(J)$ is called, for short, a partition of $J$. Let $\left\{J_{i}\right\}$ be a partition of $J$; the set of end points of the intervals $J_{i}$ is called the mesh of the partition, and the number sup $\left|J_{i}\right|$ the norm of the partition. Let $J=[a, b[$ be nonempty and bounded, and let $B\left(x_{0}, r\right) \subset \mathbb{E}, r>0$. A function $f: J \times B\left(x_{0}, r\right) \rightarrow \mathbb{E}$ is said to be piecewise locally Lipschitzean (resp. piecewise locally $\alpha$-Lipschitzean) if $f$ is bounded and there exists a partition $\left\{J_{i}\right\} \in \mathcal{I}(J)$ of $J$ such that the restriction of $f$ to each set $J_{i} \times B\left(x_{0}, r\right)$ is locally Lipschitzean (resp. locally $\alpha$-Lipschitzean).

We shall denote by $\mathcal{L}\left(J \times B\left(x_{0}, r\right)\right)$ and $\mathcal{L}^{\alpha}\left(J \times B\left(x_{0}, r\right)\right)$ the class of all functions $f: J \times B\left(x_{0}, r\right) \rightarrow \mathbb{E}$ which are, respectively, piecewise locally Lipschitzean and piecewise locally $\alpha$-Lipschitzean.

Let $F: I \times B\left(x_{0}, r\right) \rightarrow \mathcal{B}$ be a multifunction, where $I=\left[t_{0}, T[\right.$ and $B\left(x_{0}, r\right) \subset \mathbb{E}(r>0)$. We suppose:

$\left(H_{1}\right) F$ is continuous on $I \times B\left(x_{0}, r\right)$,

$\left(H_{2}\right) F$ is bounded on $I \times B\left(x_{0}, r\right)$ by a constant $M \geq 1$,

$\left(H_{3}\right) 0<T-t_{0}<r /(2 M)$.

By a solution of (1.1) (resp. (1.2), (1.3)) we mean a Lipschitzean function $x: J \rightarrow \mathbb{E}$ defined on a nondegenerate interval $J$ containing $t_{0}$, satisfying (1.1) (resp. (1.2), (1.3)) a.e. in $J$. Set

$$
\begin{aligned}
\mathcal{M}_{F} & =\{x: I \rightarrow \mathbb{E} \mid x \text { is a solution of }(1.1)\}, \\
\mathcal{M}_{\text {ext } F} & =\{x: I \rightarrow \mathbb{E} \mid x \text { is a solution of }(1.2)\} .
\end{aligned}
$$

The space $\mathcal{M}_{F}$, endowed with the metric of uniform convergence, is complete $[8]$.

For $F$ satisfying $\left(H_{1}\right)-\left(H_{3}\right)$, set $\mathcal{S}_{F}=\left\{f \in \mathcal{L}\left(I \times B\left(x_{0}, r\right)\right) \mid f\right.$ is a selection of $F\}, \mathcal{S}_{F}^{\alpha}=\left\{f \in \mathcal{L}^{\alpha}\left(I \times B\left(x_{0}, r\right)\right) \mid f\right.$ is a selection of $\left.F\right\}$. Clearly $\mathcal{S}_{F}, \mathcal{S}_{F}^{\alpha}$ are nonempty. For $f \in \mathcal{S}_{F}^{\alpha}$, we set $K_{f}=\{x: I \rightarrow \mathbb{E} \mid x$ is a solution of (1.3) $\}. K_{f}$ is a nonempty compact subset of $\mathcal{M}_{F}$ and, if $f \in \mathcal{S}_{F}$, then $K_{f}$ is a singleton.

Proposition 2.1. Let $F$ satisfy $\left(H_{1}\right)-\left(H_{3}\right)$. Let $f \in \mathcal{S}_{F}^{\alpha}$ and $\eta>0$. Then there exists $\varrho=\varrho_{f}(\eta), 0<\varrho<r / 2$, such that if $x \in C(I, \mathbb{E})$ satisfies $\left\|x(t)-x_{0}\right\|<r$ and

$$
\left\|\int_{t_{0}}^{t}[\dot{x}(s)-f(s, x(s))] d s\right\|<\varrho \quad \text { for every } t \in I,
$$

then $x \in K_{f}+\eta B$.

Proof. Suppose the statement is not true. Then there exist $f \in \mathcal{S}_{F}^{\alpha}$, $\eta>0$, and a sequence $\left\{x_{n}\right\} \subset C(I, \mathbb{E})$, with $\left\|x_{n}(t)-x_{0}\right\|<r, t \in I$, 
satisfying for each $n \in \mathbb{N}$

$$
\left\|\int_{t_{0}}^{t}\left[\dot{x}_{n}(s)-f\left(s, x_{n}(s)\right)\right] d s\right\|<\frac{r}{2 n} \quad \text { for every } t \in I,
$$

and $x_{n} \notin K_{f}+\eta B$. By a standard argument one can prove that $\alpha\left[\left\{x_{n}(t)\right\}\right]=$ 0 for every $t \in I$. Hence the sequence $\left\{x_{n}\right\} \subset C(I, \mathbb{E})$ is compact. Let $\left\{x_{n_{k}}\right\}$ be a subsequence of $\left\{x_{n}\right\}$ converging to $x$, say. As $x \in K_{f}$, for $k$ large enough we have $x_{n_{k}} \in K_{f}+\eta B$, a contradiction. This completes the proof.

Proposition 2.2. Let $T, X$ be metric spaces. Let $G: T \times X \rightarrow \mathcal{B}$ be a continuous multifunction. Let $u_{0} \in \mathbb{E}$ be such that $u_{0} \in \operatorname{int} G(t, x)$ for every $(t, x) \in T \times \widetilde{B}\left(x_{0}, \delta\right)$, where $x_{0} \in X$ and $\delta>0$. Then there exists a locally Lipschitzean selection $g$ of $G$ satisfying $g(t, x)=u_{0}$ for every $(t, x) \in T \times \widetilde{B}\left(x_{0}, \delta\right)$.

Proof. Let $(s, z) \in T \times X$. Suppose that $d\left(z, x_{0}\right)=\delta$, where $d$ is the metric of $X$. Since $u_{0} \in \operatorname{int} G(s, z)$, and $G$ is continuous, there exists a ball $B\left((s, z), \delta_{s, z}\right) \subset T \times X$ such that $u_{0} \in G(t, x)$ for every $(t, x) \in B\left((s, z), \delta_{s, z}\right)$.

Suppose $d\left(z, x_{0}\right)>\delta$. In this case choose any $u_{s, z} \in \operatorname{int} G(s, z)$. Since $G$ is continuous there exists a ball $B\left((s, z), \delta_{s, z}\right) \subset T \times X$ not intersecting $T \times \widetilde{B}\left(x_{0}, \delta\right)$ such that $u_{s, z} \in G(t, x)$ for every $(t, x) \in B\left((s, z), \delta_{s, z}\right)$. Denote by $\mathcal{U}=\{U\}$ the family whose members are $T \times B\left(x_{0}, \delta\right)$ and each of the sets $B\left((s, z), \delta_{s, z}\right)$ constructed above. $\mathcal{U}$ is an open covering of $T \times X$. For $U \in \mathcal{U}$, set

$$
y_{U}= \begin{cases}u_{0} & \text { if } U=T \times B\left(x_{0}, \delta\right), \\ u_{s, z} & \text { if } U=B\left((s, z), \delta_{s, z}\right) .\end{cases}
$$

Let $\left\{p_{U}\right\}_{U \in \mathcal{U}}$ be a partition of unity subordinate to $\mathcal{U}[6]$. Without loss of generality we suppose that the functions $p_{U}: T \times X \rightarrow[0,1]$ are locally Lipschitzean. Now, define $g: T \times X \rightarrow \mathbb{E}$ by

$$
g(t, x)=\sum_{U \in \mathcal{U}} p_{U}(t, x) y_{U}
$$

It is straightforward to verify that $g$ is a locally Lipschitzean selection of $G$ such that $g(t, x)=u_{0}$ for every $(t, x) \in T \times \widetilde{B}\left(x_{0}, \delta\right)$. This completes the proof.

Let $\mathbb{E}^{*}$ be the topological dual of $\mathbb{E}$. Let $\left\{e_{n}\right\} \subset \mathbb{E}^{*},\left\|e_{n}\right\|=1$, be a sequence dense in the unit sphere of $\mathbb{E}^{*}$ (recall that $\mathbb{E}$ is separable and reflexive). Let $\langle\cdot, \cdot\rangle$ denote the pairing between $\mathbb{E}^{*}$ and $\mathbb{E}$. Let $F: I \times$ $B\left(x_{0}, r\right) \rightarrow \mathcal{B}$ satisfy $\left(H_{1}\right)-\left(H_{3}\right)$. Following Choquet [6] and Castaing and Valadier [5], define $\varphi_{F}: I \times B\left(x_{0}, r\right) \times \mathbb{E} \rightarrow[0,+\infty]$ by

$$
\varphi_{F}(t, x, v)= \begin{cases}\sum_{n=1}^{\infty}\left\langle e_{n}, v\right\rangle^{2} / 2^{n} & \text { if } v \in F(t, x), \\ +\infty & \text { if } v \notin F(t, x) .\end{cases}
$$


Let $\mathcal{A}$ denote the class of all continuous affine functions $a: \mathbb{E} \rightarrow \mathbb{R}$. We associate with $\varphi_{F}$ the function $\widehat{\varphi}_{F}: I \times B\left(x_{0}, r\right) \times \mathbb{E} \rightarrow[-\infty,+\infty$ [ given by $\widehat{\varphi}_{F}(t, x, v)=\inf \left\{a(v) \mid a \in \mathcal{A}\right.$ and $a(z) \geq \varphi_{F}(t, x, z)$ for every $\left.z \in F(t, x)\right\}$. Now, define the Choquet function $d_{F}: I \times B\left(x_{0}, r\right) \times \mathbb{E} \rightarrow[-\infty,+\infty[$ by

$$
d_{F}(t, x, v)=\widehat{\varphi}_{F}(t, x, v)-\varphi_{F}(t, x, v) .
$$

Some known properties of the Choquet function $d_{F}$ are collected in the following proposition (see [5], [3]).

Proposition 2.3. Let $F$ satisfy $\left(H_{1}\right)-\left(H_{3}\right)$. Then we have:

(i) For each $(t, x) \in I \times B\left(x_{0}, r\right)$ and $v \in F(t, x)$ we have $0 \leq d_{F}(t, x, v) \leq$ $M^{2}$. Moreover, $d_{F}(t, x, v)=0$ if and only if $v \in \operatorname{ext} F(t, x)$.

(ii) For each $(t, x) \in I \times B\left(x_{0}, r\right)$ the function $v \rightarrow d_{F}(t, x, v)$ is concave on $\mathbb{E}$ and strictly concave on $F(t, x)$.

(iii) $d_{F}$ is upper semicontinuous on $I \times B\left(x_{0}, r\right) \times \mathbb{E}$.

(iv) For each solution $x: I \rightarrow \mathbb{E}$ of (1.1), the function $t \rightarrow$ $d_{F}(t, x(t), \dot{x}(t))$ is nonnegative, bounded and Lebesgue measurable.

(v) If $\left\{x_{n}\right\} \subset \mathcal{M}_{F}$ converges uniformly to $x \in \mathcal{M}_{F}$, then

$$
\limsup _{n \rightarrow+\infty} \int_{I} d_{F}\left(t, x_{n}(t), \dot{x}_{n}(t)\right) d t \leq \int_{I} d_{F}(t, x(t), \dot{x}(t)) d t .
$$

3. Main result. Let $F$ satisfy $\left(H_{1}\right)-\left(H_{3}\right)$. For $\theta>0$, define

$$
\mathcal{M}_{\theta}=\left\{x \in \mathcal{M}_{F} \mid \int_{I} d_{F}(t, x(t), \dot{x}(t)) d t<\theta\right\} .
$$

Lemma 3.1. Let $F$ satisfy $\left(H_{1}\right)-\left(H_{3}\right)$. Then for every $\theta>0$ the set $\mathcal{M}_{\theta}$ is open in $\mathcal{M}_{F}$.

Proof. Let $\left\{x_{n}\right\} \subset \mathcal{M}_{F} \backslash \mathcal{M}_{\theta}$ be any sequence converging to $x \in \mathcal{M}_{F}$. By virtue of Proposition 2.3(v), we have

$$
\int_{I} d_{F}(t, x(t), \dot{x}(t)) d t \geq \limsup _{n \rightarrow+\infty} \int_{I} d_{F}\left(t, x_{n}(t), \dot{x}_{n}(t)\right) d t \geq \theta,
$$

and so $x \in \mathcal{M}_{F} \backslash \mathcal{M}_{\theta}$. Hence $\mathcal{M}_{F} \backslash \mathcal{M}_{\theta}$ is closed, completing the proof.

LEMMA 3.2. Let $F$ satisfy $\left(H_{1}\right)-\left(H_{3}\right)$. Let $f \in \mathcal{S}_{F}^{\alpha}$. Let $\eta>0$ and $\theta>0$. Then there exists $g \in \mathcal{S}_{F}$ such that

$$
K_{g} \in \mathcal{M}_{\theta} \cap\left(K_{f}+\eta B\right) .
$$

Proof. The construction of $g$ is realized in three steps. In Step $1, g$ is constructed locally on a set of the form $I_{\delta} \times B\left(x_{0}, r\right)$ for some interval $I_{\delta} \subset I$. In Step 2, $g$ is extended to the whole set $I \times B\left(x_{0}, r\right)$ and it is shown that $g \in \mathcal{S}_{F}$. In Step 3, it is proved that for such $g,(3.1)$ is satisfied. 
Let $f \in \mathcal{S}_{F}^{\alpha}, \eta>0$ and $\theta>0$. Let $\varrho=\varrho_{f}(\eta)$ correspond to $f$ and $\eta$ according to Proposition 2.1. Fix $\sigma$ with

$$
0<\sigma<\min \{\varrho, \theta\} .
$$

Denote by $\left\{L_{j}\right\} \in \mathcal{I}(I)$ a partition of $I$ associated with $f$ (according to the definition of a piecewise $\alpha$-Lipschitzean function) and let $L_{j}$ be the interval of such partition containing $t_{0}$.

Step 1 (Local construction of $g$ ). Since $f\left(t_{0}, x_{0}\right) \in F\left(t_{0}, x_{0}\right)$, by the Krein-Milman theorem there exist $v_{k} \in \operatorname{ext} F\left(t_{0}, x_{0}\right)$ and $0<\lambda_{k} \leq 1(k=$ $1, \ldots, p)$, with $\sum_{k=1}^{p} \lambda_{k}=1$, such that

$$
\left\|f\left(t_{0}, x_{0}\right)-\sum_{k=1}^{p} \lambda_{k} v_{k}\right\|<\frac{\sigma}{4|I|} .
$$

By Proposition 2.3(i), (iii), there exist $u_{k} \in \operatorname{int} F\left(t_{0}, x_{0}\right)(k=1, \ldots, p)$ such that $d_{F}\left(t_{0}, x_{0}, u_{k}\right)<\sigma /|I|$, and

$$
\left\|f\left(t_{0}, x_{0}\right)-\sum_{k=1}^{p} \lambda_{k} u_{k}\right\|<\frac{\sigma}{4|I|} .
$$

Since $f$ and $F$ are continuous at $\left(t_{0}, x_{0}\right)$, and $d_{F}$ is upper semicontinuous at $\left(t_{0}, x_{0}, u_{k}\right)$, there exists a $\delta_{0}$, with $\left[t_{0}, t_{0}+\delta_{0}\left[\subset L_{j}\right.\right.$, such that for every $(t, x) \in\left[t_{0}, t_{0}+\delta_{0}\left[\times \widetilde{B}\left(x_{0}, \delta_{0}\right)\right.\right.$ we have

$$
\begin{gathered}
\left\|f(t, x)-f\left(t_{0}, x_{0}\right)\right\| \leq \sigma /(4|I|), \\
u_{k} \in \operatorname{int} F(t, x), \quad k=1, \ldots, p, \\
d_{F}\left(t, x, u_{k}\right) \leq \sigma /|I|, \quad k=1, \ldots, p .
\end{gathered}
$$

Consider the interval $I_{\delta}=\left[t_{0}, t_{0}+\delta[\right.$, where

$$
0<\delta<\min \left\{\delta_{0} / M, \sigma /(4 M)\right\}
$$

$\left(M \geq 1\right.$ is the constant in $\left.\left(H_{2}\right)\right)$. Let $\left\{J_{k}\right\}_{k=1}^{p}$ be the partition of $I_{\delta}$ given by

$$
J_{k}=\left[t_{k-1}, t_{k}\left[, \quad t_{k}=t_{0}+\sum_{h=1}^{k} \lambda_{h} \delta, \quad k=1, \ldots, p .\right.\right.
$$

By Proposition 2.2, there exists a function $g: I_{\delta} \times B\left(x_{0}, r\right) \rightarrow \mathbb{E}$ which is a selection of $F$ on $I_{\delta} \times B\left(x_{0}, r\right)$ and, moreover, for each $k, 1 \leq k \leq p$, the restriction of $g$ to $J_{k} \times B\left(x_{0}, r\right)$ is locally Lipschitzean and satisfies

$$
g(t, x)=u_{k} \quad \text { for every }(t, x) \in J_{k} \times B\left(x_{0}, \delta_{0}\right) .
$$

Let $x: I_{\delta} \rightarrow \mathbb{E}$ be the solution of the Cauchy problem

$$
\dot{x}=g(t, x), \quad x\left(t_{0}\right)=x_{0} .
$$


We claim that

$$
\begin{gathered}
d_{F}(t, x(t), \dot{x}(t)) \leq \sigma /|I|, \quad t \in I_{\delta} \text { a.e., } \\
\left\|\int_{t_{0}}^{t_{0}+\delta}[\dot{x}(s)-f(s, x(s))] d s\right\| \leq \frac{\sigma \delta}{2|I|} .
\end{gathered}
$$

In order to prove (3.10), observe that for each $t \in I_{\delta}$ we have $\left\|x(t)-x_{0}\right\|<$ $M \delta \leq \delta_{0}$, thus

$$
(t, x(t)) \in I_{\delta} \times B\left(x_{0}, \delta_{0}\right) \quad \text { for every } t \in I_{\delta} .
$$

Then, by (3.12), (3.8) and (3.6), for almost all $t \in \operatorname{int} I_{\delta}$ we have

$$
d_{F}(t, x(t), \dot{x}(t))=d_{F}(t, x(t), g(t, x(t)))=d_{F}\left(t, x(t), u_{k}\right) \leq \sigma /|I|,
$$

and (3.10) is satisfied.

Let us prove (3.11). We have

$$
\begin{aligned}
& \left\|\int_{t_{0}}^{t_{0}+\delta}[\dot{x}(s)-f(s, x(s))] d s\right\|=\left\|\delta \sum_{k=1}^{p} \lambda_{k} u_{k}-\int_{t_{0}}^{t_{0}+\delta} f(s, x(s)) d s\right\| \\
& \leq\left\|\delta \sum_{k=1}^{p} \lambda_{k} u_{k}-\delta f\left(t_{0}, x_{0}\right)\right\|+\left\|\int_{t_{0}}^{t_{0}+\delta}\left[f(s, x(s))-f\left(t_{0}, x_{0}\right)\right] d s\right\| \\
& \leq \delta\left\|\sum_{k=1}^{p} \lambda_{k} u_{k}-f\left(t_{0}, x_{0}\right)\right\|+\int_{t_{0}}^{t_{0}+\delta}\left\|f(s, x(s))-f\left(t_{0}, x_{0}\right)\right\| d s .
\end{aligned}
$$

From this, by virtue of (3.3), (3.12), and (3.4), we have

$$
\left\|\int_{t_{0}}^{t_{0}+\delta}[\dot{x}(s)-f(s, x(s))] d s\right\|<\delta \frac{\sigma}{4|I|}+\delta \frac{\sigma}{4|I|}=\frac{\sigma \delta}{2|I|},
$$

and also (3.11) is satisfied.

S t ep 2 (Global construction of $g$ ). Denote by $\mathcal{G}$ the class of all functions $g: D_{g} \times B\left(x_{0}, r\right) \rightarrow \mathbb{E}, D_{g}=\left[t_{0}, t_{g}\left[, t_{0}<t_{g} \leq T\right.\right.$, such that:

(i) $g$ is a selection of $F$ on $D_{g} \times B\left(x_{0}, r\right)$,

(ii) $g$ is a piecewise locally Lipschitzean function,

(iii) the solution $x: D_{g} \rightarrow \mathbb{E}$ of the Cauchy problem (3.9) satisfies

$$
d_{F}(t, x(t), \dot{x}(t)) \leq \sigma /|I|, \quad t \in D_{g} \text { a.e., }
$$

(iv) $D_{g}$ admits a partition $\left\{I_{i}\right\} \in \mathcal{I}\left(D_{g}\right)$ of norm strictly less than $\sigma /(4 M)$ such that, at each mesh point $t_{i}$, we have

$$
\left\|\int_{t_{0}}^{t_{i}}[\dot{x}(s)-f(s, x(s))] d s\right\| \leq \frac{\sigma\left(t_{i}-t_{0}\right)}{2|I|} .
$$


$\mathcal{G}$ is nonempty, for the function $g: I_{\delta} \times B\left(x_{0}, r\right) \rightarrow \mathbb{E}$ constructed in Step 1 satisfies (i)-(iv). Now, let us introduce in $\mathcal{G}$ a partial order. For $g_{k}: D_{g_{k}} \times B\left(x_{0}, r\right) \rightarrow \mathbb{E}(k=1,2)$, define $g_{1} \prec g_{2}$ if and only if $t_{g_{1}} \leq t_{g_{2}}$ and the restriction of $g_{2}$ to the set $D_{g_{1}} \times B\left(x_{0}, r\right)$ is equal to $g_{1}$. Let $\left\{g_{j}\right\}_{j \in \Gamma}$ be an arbitrary chain in $\mathcal{G}$. Let $\tau=\sup \left\{t_{g_{j}} \mid j \in \Gamma\right\}$. Define $g: D_{g} \times B\left(x_{0}, r\right) \rightarrow \mathbb{E}$, where $D_{g}=\left[t_{0}, \tau\left[\right.\right.$, by $g(t, x)=g_{j}(t, x)$ if $(t, x) \in D_{g_{j}} \times B\left(x_{0}, r\right)$. Clearly $g \in \mathcal{G}$ is an upper bound of the chain $\left\{g_{j}\right\}_{j \in \Gamma}$. By Zorn's Lemma there exists in $\mathcal{G}$ a maximal element, say $g$, where $g: D_{g} \times B\left(x_{0}, r\right) \rightarrow \mathbb{E}$ and $D_{g}=\left[t_{0}, t_{g}\left[\right.\right.$. We claim that $t_{g}=T$. Suppose $t_{g}<T$. Let $x: D_{g} \rightarrow \mathbb{E}$ be the solution of the Cauchy problem (3.9). Let $u$ be the limit of $x(t)$ as $t$ tends to $t_{g}$. As in Step 1 we construct a piecewise locally Lipschitzean selection of $F$ on $\Delta \times B\left(x_{0}, r\right)$, say $h: \Delta \times B\left(x_{0}, r\right) \rightarrow \mathbb{E}\left(\right.$ where $\Delta=\left[t_{g}, t_{g}+\delta[\right.$ and $0<\delta<\sigma /(4 M))$, such that the solution $y: \Delta \rightarrow \mathbb{E}$ of the Cauchy problem $\dot{y}=h(t, y), y\left(t_{g}\right)=u$, satisfies (3.10) and (3.11) (with $y, \Delta, t_{g}$ in place of $\left.x, I_{\delta}, t_{0}\right)$. Now, defining $\gamma:\left[t_{0}, t_{g}+\delta\left[\times B\left(x_{0}, r\right) \rightarrow \mathbb{E}\right.\right.$ by

$$
\gamma(t, x)= \begin{cases}g(t, x) & \text { if }(t, x) \in D_{g} \times B\left(x_{0}, r\right) \\ h(t, x) & \text { if }(t, x) \in \Delta \times B\left(x_{0}, r\right)\end{cases}
$$

one can easily see that $\gamma \in \mathcal{G}$ and $g \prec \gamma, g \neq \gamma$, a contradiction. Thus $t_{g}=T$ and the existence of a map $g: I \times B\left(x_{0}, r\right) \rightarrow \mathbb{E}$ satisfying (i)-(iv) is proved, completing Step 2.

Step 3 (The solution $x$ of (3.9) satisfies $\left.x \in \mathcal{M}_{\theta} \cap\left(K_{f}+\eta B\right)\right)$. Let $g: I \times B\left(x_{0}, r\right) \rightarrow \mathbb{E}$ satisfy (i)-(iv) (with $I$ in place of $D_{g}$ ). By construction $g \in \mathcal{S}_{F}$. Let $x: I \rightarrow \mathbb{E}$ be the solution of (3.9). From (3.13) and (3.2), we have

$$
\int_{I} d_{F}(t, x(t), \dot{x}(t)) d t<\theta
$$

thus $x \in \mathcal{M}_{\theta}$. Now, let $t \in I$. With the notations of (iv) for some mesh point $t_{i}$ of the partition $\left\{I_{i}\right\} \in \mathcal{I}(I)$, we have $\left|t-t_{i}\right|<\theta /(4 M)$. From this inequality and (3.14) it follows that

$$
\begin{aligned}
\| \int_{t_{0}}^{t}[\dot{x}(s) & -f(s, x(s))] d s \| \\
& \leq\left\|\int_{t_{0}}^{t_{i}}[\dot{x}(s)-f(s, x(s))] d s\right\|+\left\|\int_{t_{i}}^{t}[\dot{x}(s)-f(s, x(s))] d s\right\| \\
& \leq \frac{\sigma\left(t_{i}-t_{0}\right)}{2|I|}+\left|t-t_{i}\right| 2 M<\frac{\sigma}{2}+\frac{\sigma}{2}=\sigma .
\end{aligned}
$$

As the last inequality is satisfied for arbitrary $t \in I$ and $\sigma<\varrho$ (by (3.2)), Proposition 2.1 implies that $x \in K_{f}+\eta B$. Hence $x \in \mathcal{M}_{\theta} \cap\left(K_{f}+\eta B\right)$ and thus $K_{g} \in \mathcal{M}_{\theta} \cap\left(K_{f}+\eta B\right)$, for $K_{g}=x$. This completes the proof. 
Theorem 3.3. Let $F$ satisfy $\left(H_{1}\right)-\left(H_{3}\right)$. Let $f \in \mathcal{S}_{F}^{\alpha}$. Then for every $\eta>0$ we have

$$
\mathcal{M}_{\text {ext } F} \cap\left(K_{f}+\eta B\right) \neq \emptyset .
$$

In particular, $\mathcal{M}_{\operatorname{ext} F}$ is nonempty.

Proof. Fix $f \in \mathcal{S}_{F}^{\alpha}, \eta>0$ and set $\theta_{n}=1 / n(n \in \mathbb{N})$. We denote by $B(u, r)$ and $\widetilde{B}(u, r)$ an open and a closed ball in the space $\mathcal{M}_{F}$. By Lemma 3.2 there exists $g_{1} \in \mathcal{S}_{F}$ such that $K_{g_{1}} \in \mathcal{M}_{F} \cap\left(K_{f}+\eta B\right)$ and thus, for some $0<\eta_{1}<\theta_{1}$ we have

$$
\widetilde{B}\left(K_{g_{1}}, \eta_{1}\right) \subset \mathcal{M}_{F} \cap\left(K_{f}+\eta B\right) .
$$

By Lemma 3.2 there exists $g_{2} \in \mathcal{S}_{F}$ such that $K_{g_{2}} \in \mathcal{M}_{\theta_{1}} \cap B\left(K_{g_{1}}, \eta_{1}\right)$. Since, by Lemma 3.1, this set is open in $\mathcal{M}_{F}$, there exists $0<\eta_{2}<\theta_{2}$ such that

$$
\widetilde{B}\left(K_{g_{2}}, \eta_{2}\right) \subset \mathcal{M}_{\theta_{1}} \cap B\left(K_{g_{1}}, \eta_{1}\right) .
$$

Continuing in this way gives a decreasing sequence of closed balls $\widetilde{B}\left(K_{g_{n}}, \eta_{n}\right)$ $\subset \mathcal{M}_{F}$, where $g_{n} \in \mathcal{S}_{F}$ and $0<\eta_{n}<\theta_{n}$, with diameters tending to zero, satisfying

$$
\widetilde{B}\left(K_{g_{n+1}}, \eta_{n+1}\right) \subset \mathcal{M}_{\theta_{n}} \cap B\left(K_{g_{n}}, \eta_{n}\right), \quad n \in \mathbb{N} .
$$

As $\mathcal{M}_{F}$ is complete, by Cantor's intersection theorem there is one (and only one) point, say $x$, lying in all the balls $\widetilde{B}\left(K_{g_{n}}, \eta_{n}\right)$. Since $x \in \mathcal{M}_{\theta_{n}}, n \in \mathbb{N}$, we have

$$
\int_{I} d_{F}(t, x(t), \dot{x}(t)) d t=0 .
$$

Thus, by Proposition 2.3(i), $\dot{x}(t) \in \operatorname{ext} F(t, x(t))$ a.e., showing that $x \in$

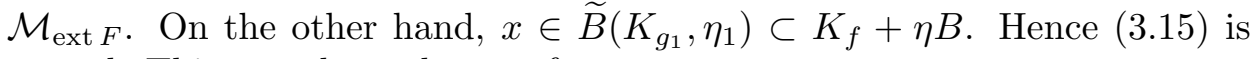
proved. This completes the proof.

\section{References}

[1] A. Antosiewicz and A. Cellina, Continuous selections and differential relations, J. Differential Equations 19 (1975), 386-398.

[2] J. P. Aubin and A. Cellina, Differential Inclusions, Springer, Berlin 1984.

[3] S. Bahi, Quelques propriétés topologiques de l'ensemble des solutions d'une classe d'équations différentielles multivoques (II), Séminaire d'Analyse Convexe, Montpellier, 1983, exposé No. 4.

[4] A. Bressan and G. Colombo, Generalized Baire category and differential inclusions in Banach spaces, J. Differential Equations 76 (1988), 135-158.

[5] C. Castaing and M. Valadier, Convex Analysis and Measurable Multifunctions, Lecture Notes in Math. 580, Springer, Berlin 1977.

[6] G. Choquet, Lectures on Analysis, Benjamin, Reading 1969. 
[7] P. V. Chuong, Un résultat d'existence de solutions pour des équations différentielles multivoques, C. R. Acad. Sci. Paris 301 (1985), 399-402.

[8] F. S. De Blasi and G. Pianigiani, A Baire category approach to the existence of solutions of multivalued differential equations in Banach spaces, Funkcial. Ekvac. (2) 25 (1982), 153-162.

[9] -, 一, The Baire category method in existence problems for a class of multivalued differential equations with nonconvex right hand side, ibid. 28 (1985), 139-156.

[10] - - -, Differential inclusions in Banach spaces, J. Differential Equations 66 (1987), $208-229$.

[11] A. F. Filippov, The existence of solutions of generalized differential equations, Math. Notes 10 (1971), 608-611.

[12] A. N. Godunov, Peano's theorem in Banach spaces, Funktsional. Anal. i Prilozhen. 9 (1) (1974), 59-60 (in Russian).

[13] H. Kaczyński and C. Olech, Existence of solutions of orientor fields with nonconvex right hand side, Ann. Polon. Math. 29 (1974), 61-66.

[14] N. S. Papageorgiou, On the solution set of differential inclusions in Banach spaces, Appl. Anal. 25 (1987), 319-329.

[15] -, On measurable multifunctions with applications to random multivalued equations, Math. Japon. 32 (1987), 437-464.

[16] G. Pianigiani, On the fundamental theory of multivalued differential equations, J. Differential Equations 25 (1977), 30-38.

[17] A. A. Tolstonogov, On differential inclusions in Banach spaces, Soviet Math. Dokl. 20 (1979), 186-190.

[18] A. A. Tolstonogov and I. A. Finogenko, On solutions of a differential inclusion with lower semicontinuous nonconvex right-hand side in a Banach space, Math. USSR-Sb. 53 (1986), 203-231.

DIPARTIMENTO DI MATEMATICA

UNIVERSITÀ DI ROMA II

VIA FONTANILE DI CARCARICOLA

00133 ROMA, ITALY
ISTITUTO DI MATEMATICA UNIVERSITÀ DI SIENA VIA DEL CAPITANO 15 53100 SIENA, ITALY 